\title{
36-year-old man with sudden severe headache
}

\author{
Paolo Simoni • Benoît Meunier • Manuel Deprez • \\ Tudor Racaru • Didier Martin
}

Received: 22 December 2010/Revised: 5 May 2011 / Accepted: 11 May 2011 /Published online: 28 May 2011

(C) ISS 2011

\section{Part I: Question}

A 36-year-old man presented to the emergency room with a complaint of severe headache that persisted for several hours. He had been suffering from unremitting, low-intensity headaches, treated with nonsteroidal anti-inflammatory drugs. The patient denied experiencing any previous brain trauma. Computed tomography (CT) without contrast administration revealed an expansive mass in the skull, centered on the left parietal bone.
The mass was inhomogeneous with a mean density of 12 HU. Higher-density linear strands, without enhancement after administration of contrast medium, were visible at the periphery of the mass. The outer table of the skull was thin but continuous, whereas the inner table was barely visible. The mass displaced the contiguous parietal and frontal lobes of the brain (Fig. 1).

At MRI, two distinct lesions were present: an inhomogeneous intradiploic mass and, inferior to this mass, an arachnoid cyst of the middle fossa of the brain. $\mathrm{T} 2 *$-weighted sequences showed no traces of bleeding within the intradiploic mass (Fig. 2).

The diagnosis can be found at doi:10.1007/s00256-011-1205-4.

P. Simoni $(\bowtie) \cdot$ B. Meunier

Department of Diagnostic Imaging,

University Hospital of Liège (CHU) Domain du Sart Tilman,

Bat. 35, 4000 Liège, Belgium

e-mail: Paolo.Simoni@chu.ulg.ac.be

\section{Deprez}

Department of Pathology,

University Hospital of Liège (CHU) Domain du Sart Tilman,

Liège, Belgium

T. Racaru $\cdot$ D. Martin

Department of Neurosurgery,

University Hospital of Liège (CHU) Domain du Sart Tilman,

Bat. 35, 4000 Liège, Belgium 


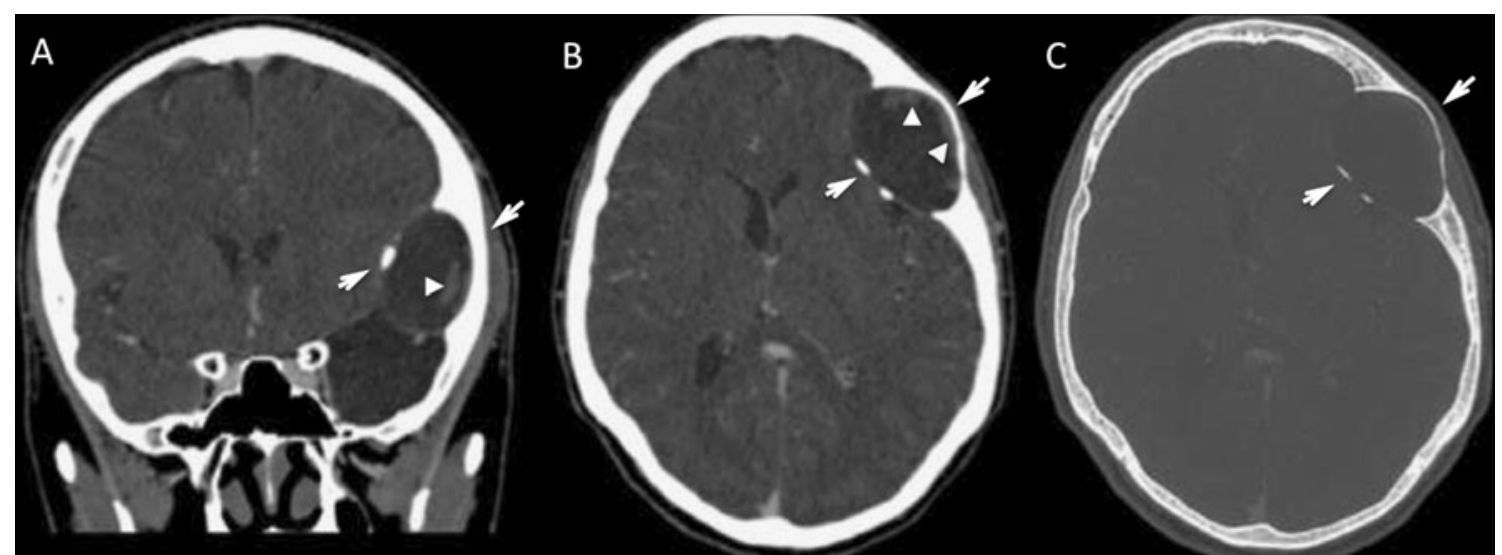

Fig. 1 Computed tomography: a reformatted coronal image of soft tissues and $\mathbf{b}, \mathbf{c}$ axial images of soft tissues and of the bone, respectively, show an expansive mass of the left parietal bone (arrows). Linear strands of higher attenuation are visible at the periphery of the mass (arrowheads)
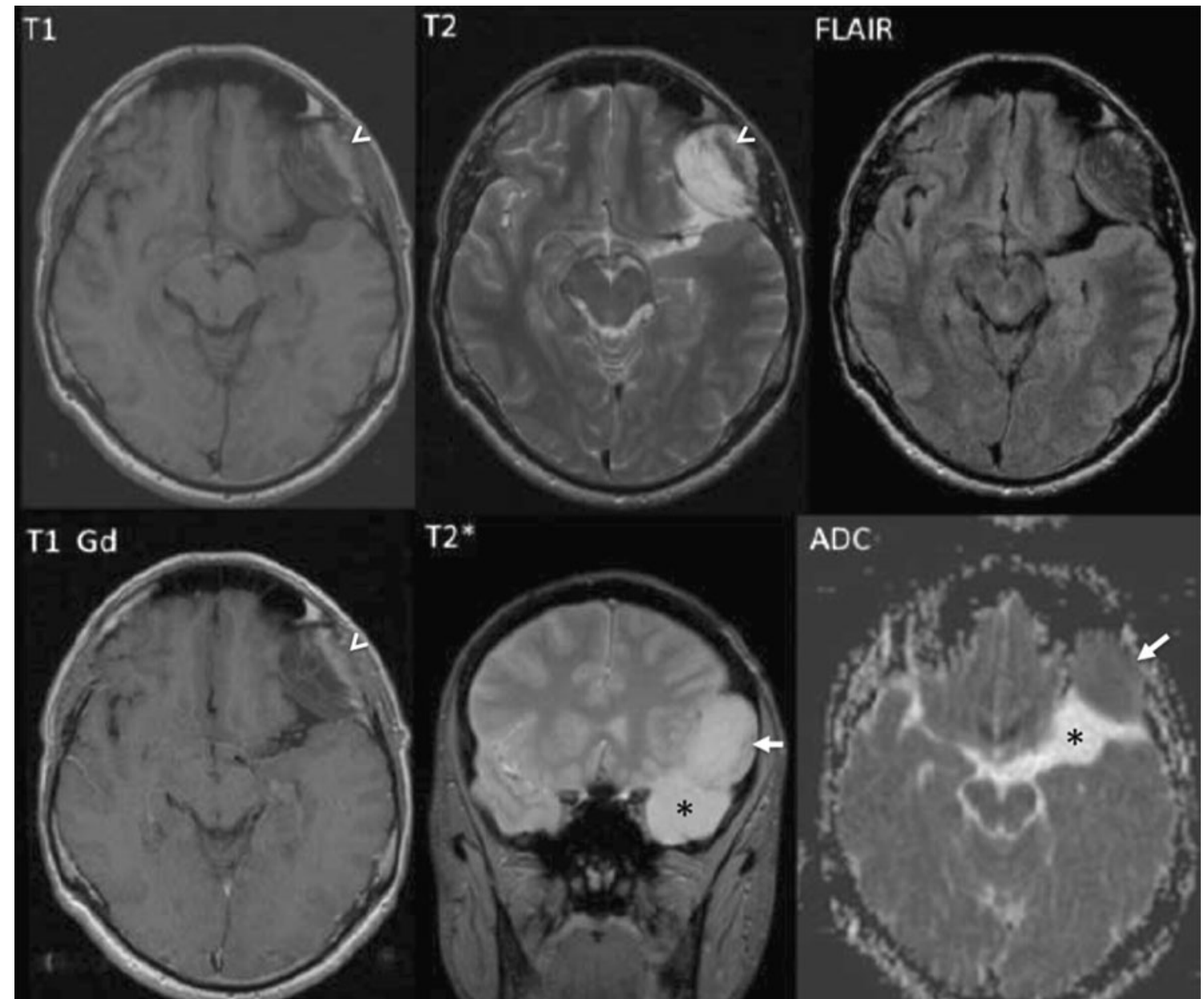

$\mathrm{T}^{*}$

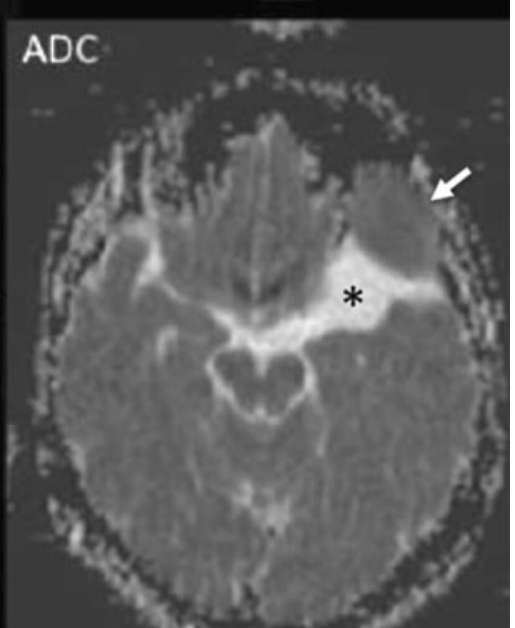

Fig. 2 MRI of a mass of the left parietal bone (arrow), displacing the contiguous brain parenchyma. Some areas with a higher signal are visible at the periphery of the mass (arrowhead). T2*-weighted coronal and axial ADC images reveal a second mass (asterisk) with a high

signal for both sequences, located inferiorly to the skull mass, consistent with cerebrospinal fluid (CSF). Note that the signal of the skull mass is similar to that of the brain parenchyma on the ADC image 